\title{
SILICA-SCALED CHRYSOPHYTES FROM ECUADOR
}

\section{CRISOFITAS ESCAMOSAS DE ECUADOR}

\author{
Daniel E. Wujek \& Ryan M. Dziedzic \\ Department of Biology, Central Michigan University, Mount Pleasant, MI 48859 USA \\ wujek1de@cmich.edu
}

\begin{abstract}
Thirty silica-scaled chrysophytes, from 60 Ecuadorian water bodies, were observed using transmission and scanning electron microscopy. The taxa comprised 27 Synurophyceae (19Mallomonas spp., 7 Synura spp., and one Chrysodidymus species) and three Chrysophyceae (two species of Paraphysomonas and one species of Spiniferomonas). All are new reports for Ecuador, and one taxon (Mallomonas lychenensis f. ecuadorensis) is described as new. The number of taxa per sample varied from none to ten.
\end{abstract}

KEYwords: Chrysophyceae, South America, Synuraceae.

\section{RESUMEN}

Treinta crisófitas escamosas, obtenidos de 60 cuerpos de agua en Ecuador, fueron observados mediante microscopía electrónica. Los taxones comprenden 27 Synuraceae (19 Mallomonas spp., siete Synura spp., y una especie de Chrysodidymus) y tres Chrysophyceae (dos Paraphysomonas spp. y una especie de Spiniferomonas). Todas son nuevos informes para Ecuador y un taxon (Mallomonas lychenensis f. ecuadorensis) se describe como nuevo. El número de taxones por muestra varió de ninguno a diez.

Palabras clave: América del Sur, Chrysophyceae, Synuraceae.

\section{INTRODUCTION}

The Chrysophyceae and Synurophyceae, characterized by an exogenous siliceous envelope composed of scales and/or bristles have long been recognized as important indicators of environmental conditions. These silica-scaled chrysophytes are second in importance only after diatoms as indicators of the past ecology of lacustrine environments ( $\mathrm{Smol}$ 1995; Stoermer 1999). For example, the effects of acid rain were demonstrated by examining present and past sediments for scaled chrysophytes to demonstrate changes in the environment over time (Smol 1995). Identifications of scaled chrysophytes are based upon the morphology of scales that often are preserved in sediments. Identifications based on light microscopy are of limited value as electron microscopy (EM) is usually necessary to distinguish sufficient morphology for species identification in the two groups (Kristiansen 1979).

Information is now becoming available on the ecology and distribution of Central and South American silica-scaled chrysophytes. An extensive literature based on EM has accumulated from several Latin American countries, especially Argentina (Vigna \& Kristiansen 2002), Brazil (Franceschini \& Kristiansen 2004), Chile (Dürrschmidt (1980, 1981, 1982a, 1982b, 1983a, 1983b, 1983c), and Costa Rica (Wujek 1984; Wujek et al. 1998). Reports from Mexico (Kristiansen \& Tong 1995), Colombia (Vigna \& Escobar 1999) and Belize (Carty \& Wujek 2003) have also been published. 
This study presents an account of scalebearing chrysophytes (Chrysophyceae and Synurophyceae) from the equatorial area of Ecuador using scanning (SEM) and transmission electron microscopy (TEM). Correlations of these organisms with ecological conditions are discussed briefly.

\section{MATERIALS AND METHODS}

Sixty different sites from Ecuador were sampled (Table I). Most sites were located using a global positioning system. Plankton net samples were taken with 10 or $20 \mu \mathrm{m}$ mesh nets. Samples were preserved with acid Lugol's solution (Gifford 1991) in plastic screw-cap vials. Collecting sites included rivers, ponds, lakes, temporarily flooded fields, and ephemeral ponds. Samples for TEM were subsampled onto Formvar-coated, carbon stabilized, $3 \mathrm{~mm}$ copper grids. After air drying, they were examined with a JEOL CM-10 TEM. Samples for scanning electron microscopy were prepared as described previously (Wujek \& Elsner 1992).

\section{RESULTS}

Thirty taxa representing five genera were observed in the 60 samples (Table II). Taxa, including one new form of Mallomonas, are all new reports for Ecuador (Figs. 1-31). The taxon list includes 19 Mallomonas taxa, seven Synura taxa, including two forms of S. petersenii, two Paraphysomonas taxa, and one taxon each of Chrysodidymus and Spiniferomonas.

The number of scale-bearing chrysophyte taxa observed per sample varied from none to ten (Table I). The most frequently observed species was Paraphysomonas vestita (Stokes) de Saedeleer (16\% of samples). This was followed by three taxa in the genus Synura: S. echinulata Korshikov (12\%), $S$. petersenii Korshikov f. petersenii $(7 \%)$ and $S$. spinosa Korshikov (5\%). Common species frequently observed from Mallomonas were $M$. matvienkoae var. matvienkoae (8\%), and three taxa each at 5\%, M. mangofera f. foveata Dürrschmidt, M. papillosa Harris \& Bradley, and M. striata var. serrata Harris \& Bradley. Mallomonas akrokomos Rutter, M. fenestrata Cronberg \& Hickel, $M$. lychenensis Conrad, M. lychenensis f.ecuadorensis
Wujek \& Dziedzic, $M$. parvula Dürrschmidt and $M$. pillula f. valdiviana Dürrschmidt, and Synura uvella Ehrenberg em. Korshikov were only observed once.

A pond with large quantities of horse dung (sample 46) had the highest diversity (10 taxa). A small marsh (sample 45) and a shallow tributary of Lake Zancudo Cocha (sample M3), whose surface was covered by a "scum" were second with seven. Over half of the sites had four or fewer species each.

One new form of Mallomonas lychenensis, M. lychenensis f.ecuadorensis, was observed (Fig. 9). The epithet "ecuadorensis" refers to the type locality.

Mallomonas lychenensis Conrad $\mathrm{f}$. ecuadorensis Wujek et Dziedzic $f$. nov.

Latin diagnosis:Differt af. lychenensis fasci scuti. Scutum 6-8 magnis lacunis irregulariter distributis. Scutum squamae areis papillarum dense obtectum. Squamae 3-3.5 x 5-6 $\mu \mathrm{m}$. Lacunae magnae (0.5-1.2 $\mu \mathrm{m}$ diametro) scutarum quasi quadrangulares tres aut quatuor seriebus duabus distributae. Nec setae nec cystae observatae.

Typus: Die 12 junii, anno 2001 in stagnum gramineum $\left(02^{\circ} 46.58^{\prime} \mathrm{S}, 7^{\circ} 13.93^{\prime} \mathrm{W}\right)$, Ecuador. Figuris 9 monstratus, in collectione Universitatis Michiganensis Centralis, Mount Pleasant, Michigan. Holotypus: Fig. 9.

Description: This species differs from $M$. lychenensis f. lychenensis in the appearance of 6-8 large, irregularly arranged pits in the shield. The shield of scales is covered with well developed patches of closely spaced papillae (Fig. 9). The scales are 3-3.5 x 5-6 $\mu \mathrm{m}$. Large pits $(0.5-1.2 \mu \mathrm{m}$ diameter) occur in two rows, three to four pits in each row. Bristles and cysts not observed.

Type locality: Pool with grass $\left(02^{\circ} 46.58^{\prime} \mathrm{S}\right.$, 79¹3.93'W), Ecuador, South America, 12 June 2001; deposited in the Central Michigan University Herbarium (CMC). Type figure: Fig. 9.

The unique size of the large pores in the shield separates this taxon from other forms. While the papillate shield area is structurally similar to $M$. lychenensis our taxon possess the very large double row of pits terminating with a large pit at the proximal end. Such a pit is lacking in f. lychenensis. It also resembles M. lychenensis f. symposiaca Skogstad \& Kristiansen, but lacks the elongated and often irregular-shaped depressions or channels along the shield. 
Ecuadorian silica-scaled chrysophytes: WuJEK \&DZIEDZIC

TABLE I. Ecuadorian site where silica-scaled chrysophytes were sampled, 1988-2001.

\begin{tabular}{|c|c|c|c|c|c|}
\hline Sample & Date & Site Description & GPS coordinates & $\mathrm{pH}$ & Taxa \\
\hline 1 & 3 June 2001 & 3 year old oxbow & $01^{\circ} 0.91^{\prime} \mathrm{S}, 77^{\circ} 0.85^{\prime} \mathrm{W}$ & 6 & 4 \\
\hline 2 & 3 June 2001 & Oxbow & $01^{\circ} 0.43^{\prime} \mathrm{S}, 77^{\circ} 2.40^{\prime} \mathrm{W}$ & 6 & 2 \\
\hline 3 & 3 June 2001 & stream & $01^{\circ} 0.41^{\prime} \mathrm{S}, 77^{\circ} 2.40^{\prime} \mathrm{W}$ & - & 2 \\
\hline 4 & 4 June 2001 & oxbow & $01^{\circ} 0.43^{\prime} \mathrm{S}, 77^{\circ} 2.40^{\prime} \mathrm{W}$ & - & 2 \\
\hline 5 & 5 June 2001 & forest pond & --- & - & 1 \\
\hline 6 & 5 June 2001 & oxbow near Nenquipare & $01^{\circ} 0.61^{\prime} \mathrm{S}, 77^{\circ} 3.48^{\prime} \mathrm{W}$ & - & 4 \\
\hline 7 & 5 June 2001 & oxbow & $01^{\circ} 0.61^{\prime} \mathrm{S}, 77^{\circ} 3.33^{\prime} \mathrm{W}$ & - & 1 \\
\hline 8 & 5 June 2001 & oxbow & $01^{\circ} 0.35^{\prime} \mathrm{S}, 77^{\circ} 3.48^{\prime} \mathrm{W}$ & - & 2 \\
\hline 9 & 6 June 2001 & swamp & $01^{\circ} 0.41^{\prime} \mathrm{S}, 77^{\circ} 3.07^{\prime} \mathrm{W}$ & 6 & 1 \\
\hline 10 & 6 June 2001 & oxbow & $01^{\circ} 0.37^{\prime} \mathrm{S}, 77^{\circ} 2.70^{\prime} \mathrm{W}$ & 6 & 1 \\
\hline 11 & 6 June 2001 & swamp & --- & - & 1 \\
\hline 12 & 7 June 2001 & pond behind dune along river & $01^{\circ} 1.49^{\prime} \mathrm{S}, 76^{\circ} 59.20^{\prime} \mathrm{W}$ & 7 & 1 \\
\hline 13 & 7 June 2001 & Schwar pond, herding corral nearby & $01^{\circ} 1.49^{\prime} \mathrm{S}, 76^{\circ} 56.92^{\prime} \mathrm{W}$ & - & 2 \\
\hline 14 & 7 June 2001 & pond by bridge over Rio Shiripuno & $01^{\circ} 0.71^{\prime} \mathrm{S}, 76^{\circ} 56.40^{\prime} \mathrm{W}$ & - & 0 \\
\hline 15 & 7 June 2001 & ditch along road near bridge & $01^{\circ} 0.51^{\prime} \mathrm{S}, 76^{\circ} 56.70^{\prime} \mathrm{W}$ & - & 1 \\
\hline 16 & 7 June 2001 & pond with floating lumber, oily & $01^{\circ} 0.42^{\prime} \mathrm{S}, 76^{\circ} 53.2 \mathrm{O}^{\prime} \mathrm{W}$ & 5.5 & 1 \\
\hline 17 & 7 June 2001 & petroleum company pond & $00^{\circ} 38.57^{\prime} \mathrm{S}, 76^{\circ} 53.13^{\prime} \mathrm{W}$ & - & 4 \\
\hline 18 & 7 June 2001 & grassy field pond & $00^{\circ} 34.58^{\prime} \mathrm{S}, 76^{\circ} 53.58^{\prime} \mathrm{W}$ & - & 2 \\
\hline 19 & 7 June 2001 & clear brown lake & --- & 5.5 & 3 \\
\hline 20 & 7 June 2001 & Pond, military camp near Coca & --- & 6 & 6 \\
\hline 21 & 9 June 2001 & Lago Cuicocha, crater lake & $00^{\circ} 17.54^{\prime} \mathrm{N}, 78^{\circ} 21.46^{\prime} \mathrm{W}$ & 7 & 3 \\
\hline 22 & 9 June 2001 & Lago San Pedro & $00^{\circ} 12.90^{\prime} \mathrm{N}, 78^{\circ} 14.27^{\prime} \mathrm{W}$ & 6.5 & 3 \\
\hline 23 & 9 June 2001 & alpine meadow, hoof prints & $00^{\circ} 11.26^{\prime} \mathrm{S}, 78^{\circ} 35.14^{\prime} \mathrm{W}$ & - & 3 \\
\hline 24 & 10 June 2001 & hotsprings & $00^{\circ} 14.03^{\prime} \mathrm{S}, 78^{\circ} 37.75^{\prime} \mathrm{W}$ & - & 1 \\
\hline 25 & 10 June 2001 & quarry puddle & $00^{\circ} 14.04^{\prime} \mathrm{S}, 78^{\circ} 36.24^{\prime} \mathrm{W}$ & - & 1 \\
\hline 26 & 12 June 2001 & Dos Chorreras trout farm & $02^{\circ} 47.07^{\prime} \mathrm{S}, 79^{\circ} 10.06^{\prime} \mathrm{W}$ & 6 & 3 \\
\hline 27 & 12 June 200 & Caballo Shayana, shallow lake & $02^{\circ} 47.23^{\prime} \mathrm{S}, 79^{\circ} 12.64^{\prime} \mathrm{W}$ & - & 0 \\
\hline 28 & 12 June 2001 & shallowpond & $02^{\circ} 47.17^{\prime} \mathrm{S}, 79^{\circ} 12.79^{\prime} \mathrm{W}$ & - & 4 \\
\hline 29 & 12 June 2001 & pond & $02^{\circ} 47.09^{\prime} \mathrm{S}, 79^{\circ} 12.87^{\prime} \mathrm{W}$ & - & 4 \\
\hline 30 & 12 June 2001 & shallow pool, rocky bottom & $02^{\circ} 47.14^{\prime} \mathrm{S}, 79^{\circ} 12.82^{\prime} \mathrm{W}$ & - & 1 \\
\hline 31 & 12 June 2001 & grassypond & $02^{\circ} 47.17^{\prime} \mathrm{S}, 79^{\circ} 12.73^{\prime} \mathrm{W}$ & 5 & 2 \\
\hline 32 & 12 June 2001 & LagoTorreodora & $02^{\circ} 46.91^{\prime} \mathrm{S}, 79^{\circ} 13.5^{\prime} \mathrm{W}$ & 6 & 2 \\
\hline 33 & 12 June 2001 & Lago Pallcacocha & $02^{\circ} 46.79^{\prime} \mathrm{S}, 79^{\circ} 13.85^{\prime} \mathrm{W}$ & 5.5 & 3 \\
\hline 34 & 12 June 2001 & pool with grass & $02^{\circ} 46.58^{\prime} \mathrm{S}, 79^{\circ} 13.93^{\prime} \mathrm{W}$ & - & 3 \\
\hline 35 & 12 June 2001 & pond & $02^{\circ} 46.59^{\prime} \mathrm{S}, 79^{\circ} 13.91^{\circ} \mathrm{W}$ & - & 1 \\
\hline 36 & 12 June 2001 & pool along road & $02^{\circ} 47.31^{\prime} \mathrm{S}, 79^{\circ} 12.98^{\prime} \mathrm{W}$ & 5.5 & 5 \\
\hline 37 & 12 June 2001 & deep pool with aquatic plants & $02^{\circ} 47.39^{\prime} \mathrm{S}, 79^{\circ} 12.82^{\prime} \mathrm{W}$ & 5.5 & 3 \\
\hline 38 & 12 June 2001 & pool & $02^{\circ} 47.38^{\prime} \mathrm{S}, 79^{\circ} 12.81^{\prime} \mathrm{W}$ & - & 2 \\
\hline 39 & 12 June 2001 & roadsidepool & $02^{\circ} 46.63^{\prime} \mathrm{S}, 79^{\circ} 14.57^{\prime} \mathrm{W}$ & - & 1 \\
\hline 40 & 13 June 2001 & pond with aquatic plants present & $02^{\circ} 54.13^{\prime} \mathrm{S}, 79^{\circ} 15.66^{\prime} \mathrm{W}$ & - & 1 \\
\hline 41 & 13 June 2001 & boggy pond, shallow, silty bottom & $02^{\circ} 54.15^{\prime} \mathrm{S}, 79^{\circ} 15.60^{\prime} \mathrm{W}$ & 5.5 & 3 \\
\hline 42 & 13 June 2001 & pond with horse dung & $01^{\circ} 54.59^{\prime} \mathrm{S}, 79^{\circ} 16.76^{\prime} \mathrm{W}$ & - & 10 \\
\hline 43 & 13 June 2001 & one of the Lagunas de Angas & $02^{\circ} 53.70^{\prime} \mathrm{S}, 79^{\circ} 17.58^{\prime} \mathrm{W}$ & - & 0 \\
\hline 44 & 13 June 2001 & lake & $02^{\circ} 53.26^{\prime} \mathrm{S}, 79^{\circ} 17.65^{\prime} \mathrm{W}$ & - & 1 \\
\hline 45 & 13 June 2001 & small marsh & $02^{\circ} 53.20^{\prime} \mathrm{S}, 79^{\circ} 17.76^{\prime} \mathrm{W}$ & - & 7 \\
\hline 46 & 13 June 2001 & small pond & $02^{\circ} 53.25^{\prime} \mathrm{S}, 79^{\circ} 17.71^{\prime} \mathrm{W}$ & 5 & 3 \\
\hline
\end{tabular}


Continuation Table I.

\begin{tabular}{|c|c|c|c|c|c|}
\hline Sample & Date & Site Description & GPS coordinates & $\mathrm{pH}$ & Taxa \\
\hline 47 & 13 June 2001 & grassymarsh & $02^{\circ} 54.15^{\prime} \mathrm{S}, 79^{\circ} 17.01^{\prime} \mathrm{W}$ & - & 1 \\
\hline 48 & 13 June 2001 & small pond & $02^{\circ} 54.14^{\prime} \mathrm{S}, 79^{\circ} 16.94^{\prime} \mathrm{W}$ & - & 0 \\
\hline 49 & 13 June 2001 & small pond & $02^{\circ} 54.13^{\prime} \mathrm{S}, 79^{\circ} 16.94^{\prime} \mathrm{W}$ & 5 & 6 \\
\hline 50 & 13 June 2001 & tiny pool between hummocks & $02^{\circ} 54.12^{\prime} \mathrm{S}, 79^{\circ} 16.98^{\prime} \mathrm{W}$ & 5 & 4 \\
\hline 51 & 15 June 2001 & park pond, Quito & $01^{\circ} 11.29^{\prime} \mathrm{S}, 78^{\circ} 29.13^{\prime} \mathrm{W}$ & - & 1 \\
\hline MI & 14-May-88 & Laguna Grande Cuyabeno, Amazonia & --- & - & 1 \\
\hline M2 & 17-May-88 & Zancudo Cocha, Amazonia & --- & - & 7 \\
\hline M3 & 17-May-88 & Zancudo Cocha, Amazonia tributary & --- & - & 7 \\
\hline M4 & 23-May-88 & Laguna Grande Cuyabeno, flooded lake & --- & - & 2 \\
\hline M5 & 29 Dec 1991 & El Junco, Galápagos & --- & - & 1 \\
\hline M6 & 13 July 1996 & Culebrilla, Andean, Cajas, stream leaving lake & --- & - & 1 \\
\hline M7 & 5 May 1993 & Temblandera, Oro Province Reservoir & --- & - & 1 \\
\hline M8 & 5 May 1993 & San Martin coastal Azolla pond & --- & - & 0 \\
\hline M9 & 24-May-88 & Texaco pond, Amazonia, Lago Agrio & --- & - & 0 \\
\hline
\end{tabular}

\section{DISCUSSION}

The scaled chrysophyte flora of Ecuador is similar to that reported for other parts of South and Central America. The species found are distributed in temperate and tropical regions (Cronberg 1989, 1996; Hansen 1996). Most surprising was the low occurrence of Synura uvella, a ubiquitous species which was observed in only one of our samples (Table II).

Mallomonas lanalhuensis (Fig. 7) is reported for only the second time since its original description from Chile (Dürrschmidt 1983a). Similarly, the occurrence of Mallomonas fenestrata (Fig. 4) from a small marsh represents the fourth report of a species to date found only in South America (Cronberg \& Hickel 1985; Cronberg 1989; Kristiansen \& Menezes 1998). Some of the other taxa that we observed were originally described from South and Central America, but have since been shown to be cosmopolitan. Examples include Mallomonas cristata, M. guttata, M. multisetigera, M. parvula and M. pillula $\mathrm{f}$. valdiviana.

The number of species in each sample was relatively low. The total is considerably less than might have been expected considering that, with only two exceptions, all of the samples came from sites where $\mathrm{pH}$ was less than 7.0 (Table I). This is surprising, because acidic conditions have been shown to be optimal for scaled chrysophyte growth (Roijackers \& Kessels 1986; Siver 1988, 1989, 1991).

Although water temperatures were not taken at time of sampling, the species observed tolerate wide ranges and are therefore considered as eurythermal (Takahashi 1978). Future South American studies should include this ecological parameter. Roijackers and Kessels (1986) have demonstrated in temperate waters the abundance of a particular species is mostly controlled by water temperature. In subtropical and tropical areas, Saha and Wujek (1990) and Wujek and Saha (1996) have suggested that where average air or water temperatures are at $20^{\circ} \mathrm{C}$ continuously, these temperatures could affect development and diversity of scaled chrysophyte populations.

This paper raises the known silica-scaled chrysophyte flora of Ecuador to a total of 30 taxa. This is far fewer than the numbers observed from Argentina (58), Brazil (52) and Chile (40). However these three countries have had their scaled chrysophyte flora studied more extensively. It is hoped our study will stimulate further Ecuadorian studies. Collections and observations representing other seasons and/or other Ecuadorian geographic regions and habitat types will undoubtedly yield additional species.

Some types of habitat have yet to be thoroughly studied worldwide, including in Ecuador. Kristiansen (1986) has stated that one such habitat type in need of research is high altitude lakes in tropical mountains. This is supported by Cronberg (1989) who states "that many more species belonging to this algal group will be found if systematicinvestigations of the tropics at different seasons of the year and over several years are undertaken". 
Ecuadorian silica-scaled chrysophytes: WuJEK \&DZIEDZIC

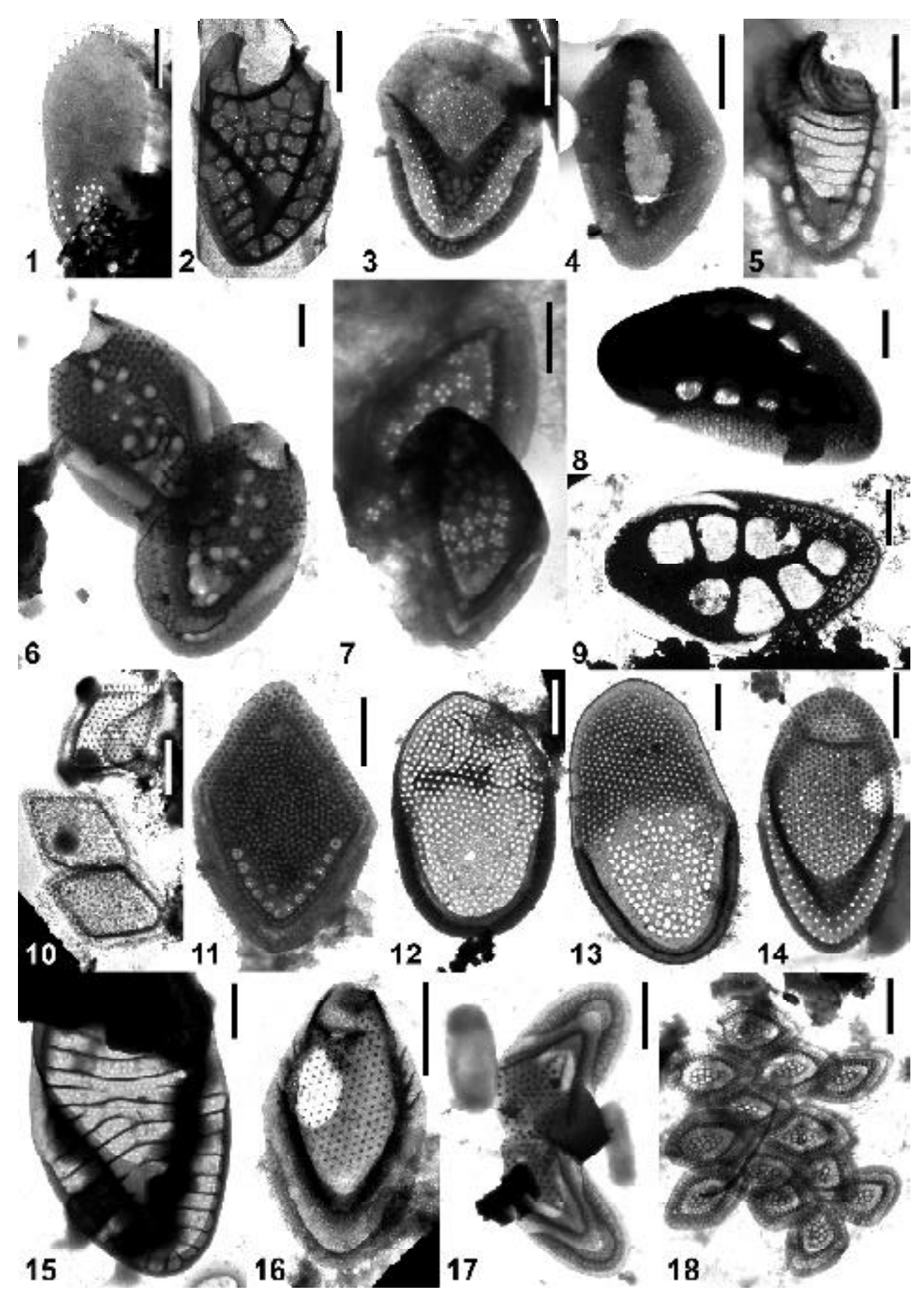

Figures. 1-18. Mallomonas. 1. M. akrokomos, slipper scale. 2. M. crassisquama, apical scale. 3. M. cristata, body scale. 4. M. fenestrata, body scale. 5. M. flora, body scale. 6. M. guttata, body scale. 7. M. lanalhuensis, body scales. 8. M. lychenensis, body scale. 9. M. lychenensis f. ecuadorensis, body scale. 10. M. mangofera f. mangofera, body scales. 11. M. mangofera f. foveata, body scale. 12. M. matvienkoae var. matvienkoae, body scale. 13. M. matvienkoae var. grandis, body scale. 14. M. multisetigera, body scale. 15. M. paludosa, body scale. 16. M. papillosa, body scale. 17. M. parvula, body scale. 18. M. pillula f. valdiviana, body scales. Scale bar $=1 \mu \mathrm{m}$. 


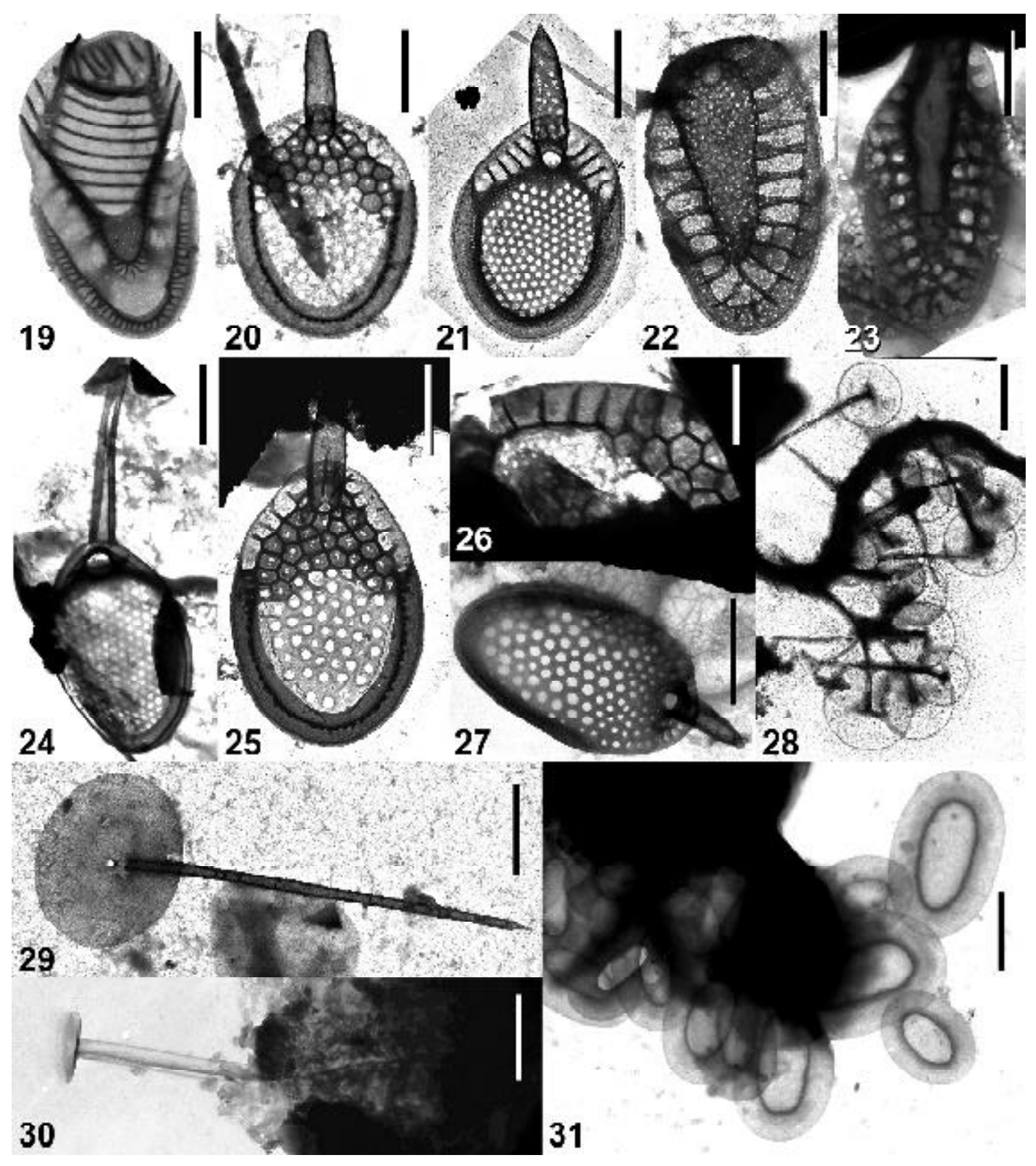

Figures 19-31. Mallomonas striata var. serrata, body scale. Figs. 20-26. Synura. 20. S. curtispina, body scale. 21. S. echinulata, body scale. 22. S. petersenii f. petersenii, body scale. 23. S. petersenii f. kufferathii, body scale. 24 . S. sphagnicola, body scale. 25. S. spinosa, body scale. 26. S. uvella, body scale. Fig. 27. Chrysodidymus synuroideus, body scale. Figs. 28-29. Paraphysomonas. 28. P. vestita, body scales. 29. P. imperforata, body scale. Figs. 30-31. Spiniferomonas trioralis. 30. Spine scale. 31. Plate scales. Scale bar $=1 \mu \mathrm{m}$. 
Ecuadorian silica-scaled chrysophytes: WuJEK \&DZIEDZIC

TABLE II. Taxa observed in the Ecuador collections. See Table I for locations.

\begin{tabular}{|c|c|}
\hline Taxon & Sites \\
\hline \multicolumn{2}{|l|}{ Synirophyceae } \\
\hline Msilanoras abohowas Rillner & 32 \\
\hline$M$ crassisqurana (Ámmd) Fott & $\mathbf{M 6}$ \\
\hline M cristata Dïnschmiat & $26, \mathrm{ML}, \mathrm{MB}$ \\
\hline M fenestrta Cronberg \& Hikel & 45 \\
\hline M flon Hamis \& Eradley & 42,47 \\
\hline$M$ guttata whjek & 17,20 \\
\hline M lanahuenis Dïnschmitt & $42,46,49$ \\
\hline M buchenensis Corrad & 42 \\
\hline 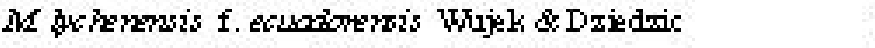 & 34 \\
\hline$M$ mongafera Hamis \& Bralleyf. mangctira & $1,4,6,13,20$ \\
\hline$M$ mongefera f. foveata Dïnschmiat & $1,6,12,17,18,20, \mathrm{ML}, \mathrm{MB}$ \\
\hline 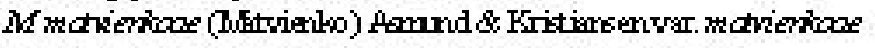 & $2,3,7,13,16,17,19,20,2123,42,45$ \\
\hline M matrienkoze var. grandis Dürschmitt \& Cronberg & 16,17 \\
\hline M multisetigera Dïnschmidt & $5,23,42,45,49$ \\
\hline M paluriasa Fott & $49,50, \mathrm{ML}, \mathrm{MB}$ \\
\hline M pronlara Hamis o Bradley & $22,31,32,34,36,41,42$ \\
\hline 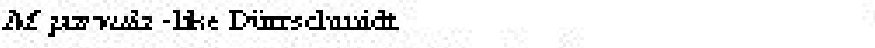 & 42 \\
\hline M prinula Haris f. valdiviana Dürrschmitt & 2 \\
\hline M striata Asmmidvar. sempta Harris \& Eradley & $16,28,29,37,41,42,45$ \\
\hline Sympacustispina (Petersen \& Hansen) Asmmd & 13,14 \\
\hline Sechimukta Kashikov & $19,20,23,28,29,30,33,37,38,41,42,45,47$, \\
\hline 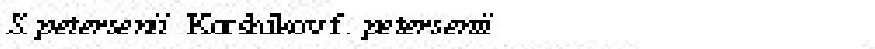 & $1,3,4,6,26,33,76,45,46,40$ \\
\hline S peterseni f hofferatini Petersen \& Hansen & $2,3,8,42$ \\
\hline S sphamic da Kordilkor & 10 \\
\hline S spinosa Konshitsos & $6,28,29,34,36,39,50, \mathrm{ml}$ \\
\hline S. uve Ila Elrenbergem. Korshkov & 26 \\
\hline $\begin{array}{l}\text { Copysadidimus spmuticieus Prowse } \\
\text { CDryscplyceae }\end{array}$ & $31,48, \mathrm{M}, \mathrm{MB}$ \\
\hline Parapisomanas imgerforata Lucas & 22,36 \\
\hline P. vestitz (Sokes) de Saedeleer & $\begin{array}{l}6,10,11,15,16,18,20,22,24,25,28,33,35 \\
36,37,40,42,44,49,51, \mathrm{MB}, \mathrm{MB}, 1 \mathrm{~J}\end{array}$ \\
\hline Syingerawona triorabs Thkshashi & 7,16 \\
\hline
\end{tabular}

\section{ACKNOWLEDGMENTS}

We thank Drs. Susan Carty and Miriam Kennan for the samples, Dr. Jørgen Kristiansen for examining several of our micrographs, Geoffrey Williams for the preparation of the carbonstabilized grids, and G Williams and Amanda Weber with the figures. This study was supported by a CMU FRCE Award.

\section{BIBLIOGRAPHY}

Carty, S. \& D.E. Wujek. 2003. A new species of Peridinium and new records of dinoflagellates and silica-scaled chrysophytes from Belize. Caribbean Journal of Science 39:136-139.

Cronberg, G. 1989. Biogeographical studies on scaled chrysophytes from the tropics. Nova Hedwigia 95: 191-232.

Cronberg, G. 1996. Scaled chrysophytes from the Okavango Delta, Botswana, Africa. Beihefte zur Nova Hedwigia 114: 91-108.

Cronberg, G. \& B. Hickel. 1985. Mallomonas fenestrata sp. nov. and M. perforata sp. nov. (Chrysophyceae. Mallomonadaceae) from tropical lakes. Nordic Journal of Botany 5: 105110.

DüRrschmidT, M.1980. Studies on the Chrysophyceae from Rio Cruces, Prov. Valdivia, South Chile, by scanning and transmission electron microscopy. Nova Hedwigia 33: 353-388. 
DüRRSCHMIDT, M. 1981. Mallomonas cristata sp. nov. (Chrysophyceae, Synuraceae) from south Chilean inland waters. Phycologia 20: 298-302.

DürRschmidt, M. 1982a. Studies on the Chrysophyceae from south Chilean inland waters by means of scanning and transmission electron microscopy, 2. Algological Studies 31: 121-163.

DürRschmidt, M. 1982b. Mallomonas parvula sp. nov. and Mallomonas retifera (Chrysophyceae, Synuraceae) from south Chile. Canadian Journal of Botany 60: 651-656.

Dürrschmidt, M. 1983a. Three new species of Mallomonas (Chrysophyceae, Mallomonadaceae) from Lake Lanalhue, Chile. Nordic Journal of Botany 3: 423-430.

DürRschmidt, M. 1983b. A taxonomic study of Mallomonas mangofera group (Synuraceae, Chrysophyceae) including the description of four new taxa. Plant Systematic and Evolution 143: 175-196.

DürRsChMidT, M. 1983c. New taxa of the genus Mallomonas (Mallomonadaceae, Chrysophyceae) from southern Chile. Nova Hedwigia 38: 717-726.

Franceschini, I.M. \& J. KRistiansen. 2004. New records of scale-bearing chrysophytes for Florianópolis, Santa Catarina Island, Southern Brazil. Algological Studies 111: 63-77.

GifFord, D.J. 1991. The protozoan-metazoan link in pelagic ecosystems. Journal of Protozoology 38: 81-86.

Hansen, P. 1996 Silica-scaled Chrysophyceae and Synurophyceae from Madagascar. Archiv für Protistenkunde 147: 145-172.

Kristiansen, J. 1979. Problems in classification and identification of Synuraceae (Chrysophyceae). Schweizerische Zeitschrift für Hydrologie 40: 310-319.

Kristiansen, J. 1986. Identification, ecology, and distribution of silica-scale-bearing Chrysophyceae, a critical approach. In: Chrysophytes: aspects and problems (eds J. Kristiansen \& R.A. Andersen), pp. 229-239. Cambridge University Press, Cambridge.

Kristiansen, J. \& M. Menezes. 1998. Silica-scaled chrysophytes from an Amazonian flood-plain lake, Mussurá, northern Brazil. Algological Studies 90: 97-118.

Kristiansen, J. \& D. Tong. 1995. A contribution to the knowledge of the silica-scaled chrysophytes in Mexico. Archiv für Hydrobiologie 77: 1-6.

RoIJACKers, R.M.M. \& H. Kessels. 1986. Ecological characteristics of scale-bearing Chrysophyceae from the Netherlands. Nordic Journal of Botany 6: 373-385.

SAHA, L.C. \& D.E. Wujek. 1990. Scaled-chrysophytes from northeast India. Nordic Journal of Botany 10: $343-354$

Siver, P.A. 1988. Distribution of scaled chrysophytes in 17 Adirondack (New York) lakes with special reference to $\mathrm{pH}$. Canadian Journal of Botany 66: 1391-1403.

Siver, P.A. 1989. The distribution of scaled chrysophytes along a $\mathrm{pH}$ gradient. Canadian Journal of Botany 67:2120-2130.

Siver, P.A. 1991. The Biology of Mallomonas: Morphology, Taxonomy and Ecology. Kluwer, The Netherlands. $230 \mathrm{pp}$.

SMoL, J.P. 1995. Applications of chrysophytes to problems in paleoecology. In: Chrysophytes Algae: Ecology, Phylogeny and Development (eds. C. Sandgren, J.P. Smol \& J. Kristiansen), pp. 303-329. Cambridge University Press, Cambridge.

Stoermer, E.F. 1999. The Diatoms: Applications for the Environmental and Earth Sciences. Cambridge University Press, Cambridge. 469 pp.

TAKAHASHI, E. 1978. Electron microscopical studies of the Synuraceae (Chrysophyceae) in Japan. Tokai University Press, Tokyo. $194 \mathrm{pp}$.

Vigna, M.S. \& S.D. Escobar. 1999. Silica-scaled chrysophytes from the Amazonian region in Colombia. Nova Hedwigia 69: 151-162.

Vigna, M.S. \& J. KRistiansen. 2002. Chrysophyceae y Synurophyceae de Tierra del Fuego. Monografias del Museo Argentino de Ciencias Naturales, $\mathrm{N}^{\circ}$ 9. $45 \mathrm{pp}$.

Wujer, D.E. 1984. Scale-bearing Chrysophyceae (Mallomonadaceae) from North-Central Costa Rica. Brenesia 22: 309-313.

Wujer, D.E. \& P.R. Elsner. 1992. Pterocystis ebelii, a new heliozoa from India. Transactions of the American Microscopical Society 111: 143-148.

Wujek, D.E. \& L.C. SAha. 1996. Scale bearing chrysophytes from India. II. Beihefte Nova Hedwigia 112: 365-375.

Wujek, D.E., R.E. Clancy, JR. \& S.P. Horn. 1998. Silica scaled Chrysophyceae and Synurophyceae from Costa Rica. Brenesia 49-50: 11-19. 